\title{
Effect of majority carrier space charges on minority carrier injection in dye doped polymer light-emitting devices
}

Cite as: Journal of Applied Physics 83, 4403 (1998); https://doi.org/10.1063/1.367200

Submitted: 26 August 1997 . Accepted: 05 January 1998 . Published Online: 07 July 1998

S. Berleb, W. Brütting, M. Schwoerer, R. Wehrmann, and A. Elschner

ARTICLES YOU MAY BE INTERESTED IN

Carrier tunneling and device characteristics in polymer light-emitting diodes

Journal of Applied Physics 75, 1656 (1994); https://doi.org/10.1063/1.356350

Relationship between electroluminescence and current transport in organic heterojunction light-emitting devices

Journal of Applied Physics 79, 7991 (1996); https://doi.org/10.1063/1.362350

Organic electroluminescent diodes

Applied Physics Letters 51, 913 (1987); https://doi.org/10.1063/1.98799

\section{Lock-in Amplifiers up to $600 \mathrm{MHz}$}
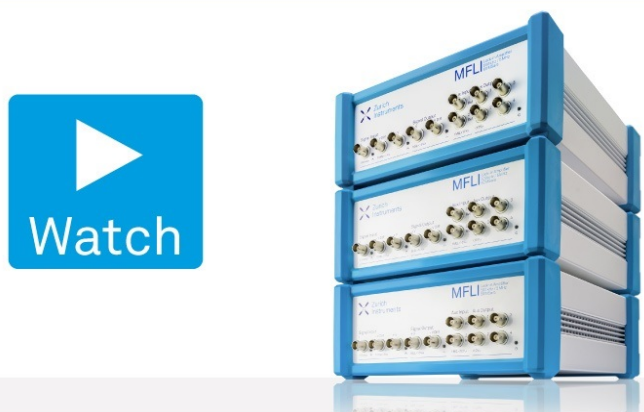


\title{
Effect of majority carrier space charges on minority carrier injection in dye doped polymer light-emitting devices
}

\author{
S. Berleb, W. Brütting, ${ }^{\text {a) }}$ and M. Schwoerer \\ Experimentalphysik II, Universität Bayreuth, 95440 Bayreuth, Germany \\ R. Wehrmann and A. Elschner \\ Bayer AG, Zentrale Forschung und Entwicklung, Werk Uerdingen, 47829 Krefeld, Germany
}

(Received 26 August 1997; accepted for publication 5 January 1998)

\begin{abstract}
By blending suitable dyes into poly(N-vinylcarbazole) we have fabricated devices which emit light in the whole visible spectrum. Their current-voltage characteristics can be described by space-charge limited currents with effective trapping of the charge carriers by the dye molecules, while the light intensity shows a Fowler-Nordheim-like behavior as a function of the external electric field. However, the anodic space charge changes the field distribution inside the device and leads to a cathodic field enhancement which has to be considered in the Fowler-Nordheim equation. We were able to model the electroluminescence characteristics by assuming tunneling of the minority charge carriers through a triangular barrier. The obtained barrier heights showed a strong dependence on the dye molecules, suggesting that the injection of minority charge carriers takes place directly into the lowest unoccupied molecular orbital of the chromophors. Using poly(p-phenylenevinylene) and an oxadiazole starburst molecule as additional hole and electron transport layers, respectively, luminance of $250 \mathrm{~cd} / \mathrm{m}^{2}$ and external quantum efficiency of $1 \%$ could be achieved. (C) 1998 American Institute of Physics. [S0021-8979(98)01808-8]
\end{abstract}

\section{INTRODUCTION}

The use of molecular dyes blended with polymer matrices is a widely used approach to the color tuning of organic light-emitting devices. Emission over the whole visible range and also white light emission have been achieved. ${ }^{1-7}$ The aim of this approach is to separately optimize the electrical and optical properties by suitable matrices and dyes. Often the assumption is made that the electrical properties (charge carrier injection and transport) are determined solely by the matrix, whereas the dye is responsible for the emission characteristics. However, this does only hold for low dye contents. Additionally, the description of the device characteristics in the rigid band model, ${ }^{8}$ where the barriers for charge carrier injection are simply given by the differences of the metal work functions and the energy levels (highest occupied and lowest unoccupied molecular orbital, HOMO and LUMO) of the organic material, is not satisfactory. The assumption of a homogeneous voltage drop over the device is no longer valid in the presence of space charges. Instead of rigid bands one has to take into account a spatially nonuniform electric field distribution. It has already been shown that in two-layer devices consisting of a hole transport layer and an electron transport layer space charges lead to field enhancement at the interface which promotes charge carrier injection through the barrier. ${ }^{9}$

In this paper we present investigations of currentvoltage and brightness-voltage characteristics showing that different dyes together with space-charge limited currents (SCLC) of holes have significant influence on electron injection already in single-layer devices. Additionally two- and

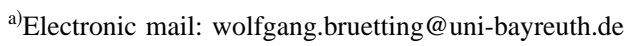

three-layer devices with poly(p-phenylenevinylene) (PPV) as hole transport layer and an oxadiazole starburst molecule as electron transport layer are investigated.

\section{MATERIALS AND EXPERIMENT}

Several fluorescent dyes blended with poly $(\mathrm{N}-$ vinylcarbazole) (PVK) were investigated for their possible use in light-emitting devices (see Figure 1): low-molecular weight coumarine (1), (2) and methine (3) dyes and a PVKderivated side chain polymer with a naphthalimide luminophore (4). The composition of the blends was 30/70 wt $\%$ of the dye in PVK, except in the case of dye (2) with a dye content of only $15 \mathrm{wt} \%$. Although one has to expect fluorescence quenching with such high dye concentrations, at the given values the highest electroluminescence yields were achieved in single-layer devices (compare also Ref. 10). This already indicates that the dye molecules have an influence on the electrical properties of the devices.

The polymer films were prepared by spin-coating of a solution of the dye/PVK blend (1\% in dichlorethane) to obtain films of typically $100 \mathrm{~nm}$ thickness. For higher film thickness up to $300 \mathrm{~nm}$ a precision doctor-blade technique was used. In the single-layer configuration the electroluminescent devices were fabricated with the polymer layer sandwiched between a transparent indium tin oxide (ITO) and an $\mathrm{Al}$ or $\mathrm{Ca}$ electrode. For two-layer devices a spin-coated 50 nm PPV film (prepared by the precursor route) was introduced as hole transport material between ITO and the PVK/ dye blend. In the three-layer configuration an additional electron transport layer consisting of a $30 \mathrm{~nm}$ thick layer of an oxadiazole starburst molecule ${ }^{11}$ was vacuum-evaporated on 
<smiles>CNc1ccc2cc(-c3nc4cc(C)ccc4o3)c(=O)oc2c1</smiles>

(1)<smiles>N#CC(C#N)=Cc1ccc(N(c2ccccc2)c2ccccc2)cc1</smiles>

(3)

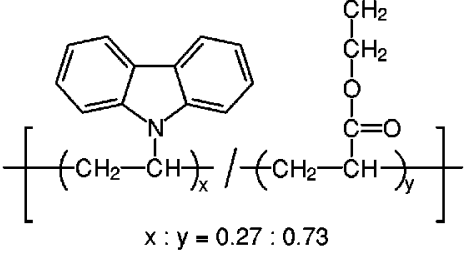

(4)

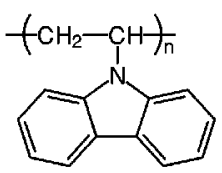

PVK

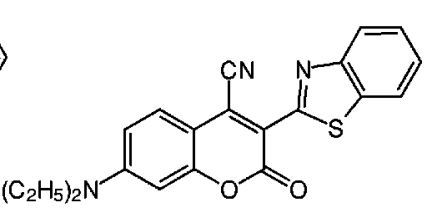

(2)

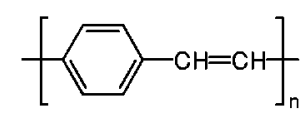

PPV

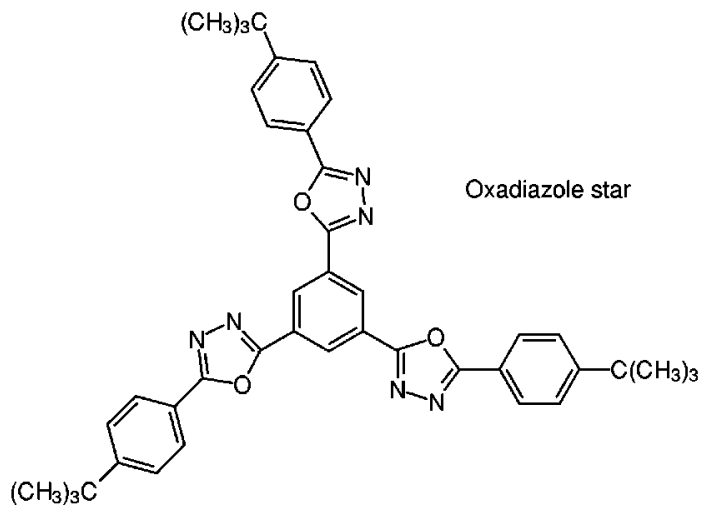

FIG. 1. Chemical structure of the materials used for organic light-emitting devices: fluorescent dyes (1)-(4) used in blends with PVK, PPV as hole transport material and an oxadiazole starburst molecule as electron transport material.

top of the PVK/dye layer. The structures of PPV and the oxadiazole are also given in Figure 1.

Current-voltage $(I-V)$ characteristics of the devices were recorded using a computer controlled source-measure unit (Keithley 236). Simultaneously with the current the flux of emitted light was measured using an integrating sphere with a calibrated Si photodiode and an electrometer (Keithley 617). The external quantum efficiency was obtained as the total emitted light power divided by the current and the average photon energy (determined from the emission spectrum). Emission spectra were obtained by a liquid nitrogen cooled charge coupled device (CCD) detector coupled to a monochromator (Princeton Instruments, Solar). The Commission Internationale d'Eclairage (CIE) color coordinates

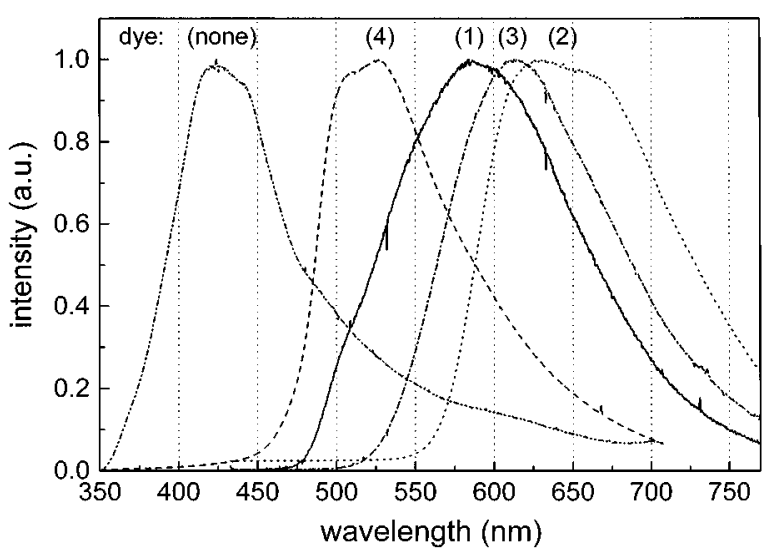

FIG. 2. Electroluminescence emission spectra of PVK and blend systems of PVK with dyes shown in Figure 1.

and luminance were taken by a spot photometer (Minolta CS-100). Film preparation and all measurements were performed under ambient conditions.

\section{SINGLE-LAYER DEVICES}

\section{A. Experimental results}

Figure 2 shows the electroluminescence (EL) emission spectra of these dyes blended with PVK. Together with the blue emission of PVK they span the whole visible range. In Table I the corresponding CIE coordinates (x, y) are listed.

In Figure 3 the typical dependence of current and brightness on the applied voltage for thick polymer layers $(d \approx 300$ $\mathrm{nm}$ ) is depicted. At low bias voltage the $I-V$ characteristics are rather symmetrical and can be described by a power law $\left(I \propto V^{n}\right)$ with an exponent between 4 and 7 depending on the dispersed dye. A kind of "dephasing" between current and voltage can be observed for bias around zero, which depends on sweep direction and speed. This can be explained in terms of trapped space charges near the injecting electrode.

At higher bias voltages $(V>30 \mathrm{~V})$, when electroluminescence starts, the current increases more steeply with the applied voltage than for low voltages. The rectification ratio is typically $10^{3}$ and higher. This behavior of the current is only observed when applying both an electron and a hole injecting contact. Using two high work function metals, e.g., ITO and Au as anode and cathode, respectively, only holes are injected and therefore neither light emission nor the steep increase of the current can be observed. Thus double injection is responsible for both the electroluminescence and the high current flow observed at high forward bias. In this regime the current is much higher than one would expect from the extrapolation of the low-bias current-voltage characteristics (see Figure 3) and follows a Fowler-Nordheim law $\left[I \propto V^{2} \exp \left(-C / V^{2}\right)\right]$ as indicated by the fit curve in Figure 3.

TABLE I. CIE coordinates of ITO/PVK + dye/Al devices.

\begin{tabular}{cccccc}
\hline \hline Dye & None & $(1)$ & $(2)$ & $(3)$ & $(4)$ \\
\hline $\mathrm{x}$ & 0.20 & 0.40 & 0.62 & 0.51 & 0.28 \\
$\mathrm{y}$ & 0.20 & 0.52 & 0.37 & 0.44 & 0.48 \\
color & blue & yellow & red & orange & green \\
\hline \hline
\end{tabular}




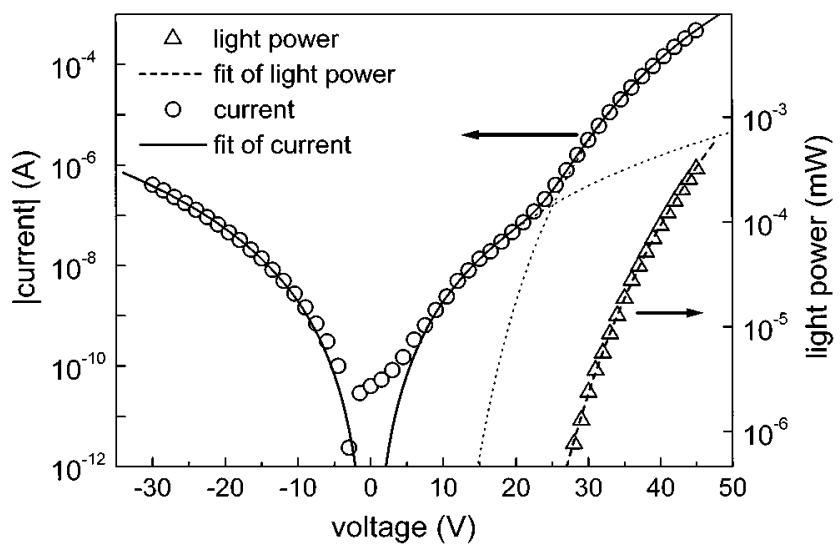

FIG. 3. Current-voltage and brightness-voltage characteristics of blend system PVK+dye(1) (film thickness $300 \mathrm{~nm}$, active area $0.25 \mathrm{~cm}^{2}$ ). The $I-V$ characteristics beetween -30 and $+20 \mathrm{~V}$ can be fitted with a power law $I \propto V^{4}$. For higher voltage it follows a Fowler-Nordheim type behavior. The combined fit of the power law and the Fowler-Nordheim law is indicated by the solid line. The light power can also be fitted by a modified Fowler-Nordheim law (dashed line), as will be discussed below.

Interestingly the brightness-voltage characteristics also show a Fowler-Nordheim-type behavior [light power $P_{E L}$ $\left.\propto V^{2} \exp \left(-C / V^{2}\right)\right]$. The dashed line in Fig. 3 indicates the fit of this expression. Originally, the Fowler-Nordheim law gives a quantitative description of the injection current density for tunneling of charge carriers from a metal into vacuum or an insulator as a function of the electric field at the contact. ${ }^{12}$ However, we will show below that in our case the Fowler-Nordheim law seen in the current is not due to tunneling of majority carriers and additionally the formulation of the Fowler-Nordheim law for tunneling of minority carriers has to be modified due to the influence of majority carrier space charges.

Generally, in these devices the onset voltage scales with the polymer layer thickness $d$ and thus the device characteristics are field-dependent and not voltage-dependent. As an example, we have plotted in Figure 4 current-voltage and brightness-voltage dependence against the applied electric field $(V / d)$ for two different film thicknesses. While the

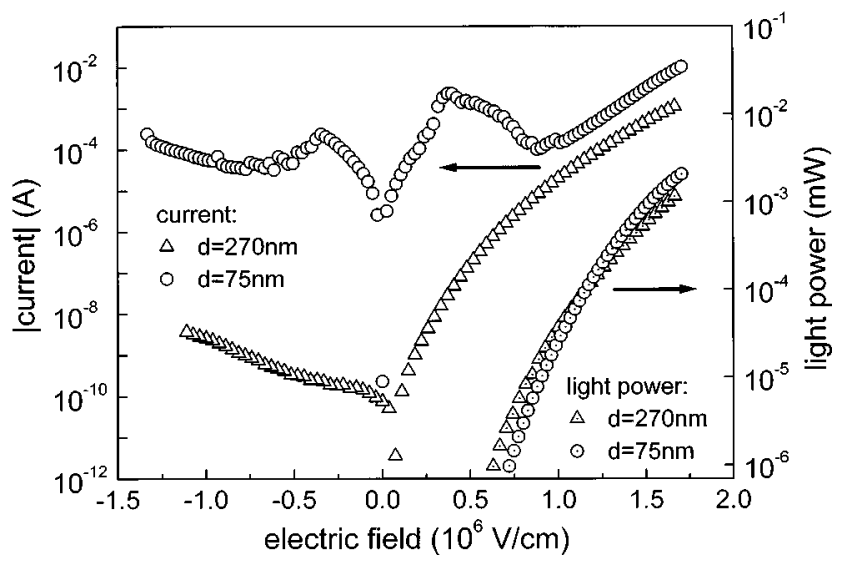

FIG. 4. Current-voltage and brightness-voltage characteristics of blend system PVK+dye(2) for two different film thicknesses $d$ (active area 0.25 $\mathrm{cm}^{2}$ ).
TABLE II. Characteristics of ITO/PVK + dye/Al devices $\left(E_{\text {onset }}\right.$ : EL onset field, $\eta_{\text {ext }}$ : maximum external quantum efficiency, $\bar{\phi}_{B}$ : effective barrier height).

\begin{tabular}{cccccc}
\hline \hline Dye & None & $(1)$ & $(2)$ & $(3)$ & $(4)$ \\
\hline$E_{\text {onset }}\left(10^{5} \mathrm{~V} / \mathrm{cm}\right)$ & 16 & 7 & 9 & 8 & 20 \\
$\eta_{\text {ext }}(\%)$ & 0.006 & 0.05 & 0.07 & 0.05 & 0.03 \\
$\bar{\phi}_{B}(\mathrm{eV})$ & 0.51 & 0.21 & 0.25 & 0.30 & 0.61 \\
\hline \hline
\end{tabular}

thick device shows a pronounced diode behavior, the current of the thin device is higher by several orders of magnitude, except for high forward bias. In spite of the large discrepancy and the occurence of local current maxima at low voltage in the thin device, the electroluminescence has almost the same onset and field dependence for both devices. In a forthcoming paper we will show that the local instable maxima in the current do not influence the brightnessvoltage characteristics. Hence the evaluation of the brightness-voltage dependence is possible also for thinner structures. However, for the quantitative evaluation of the current we have chosen rather thick polymer layers, since the appearance of these low voltage current anomalies for $d$ below about $200 \mathrm{~nm}$ causes considerable difficulties for the interpretation of the $I-V$ characteristics.

Furthermore we observe an influence of the dye blended with PVK on the onset field (see Table II) and the exponent of the power law. We note that, e.g., for dye (2) the exponent of the power law in the current (see Figure 4) is close to 7. Additionally, the change of slope in the current with the onset of EL and the Fowler-Nordheim law above this voltage is not observed with this dye. This may be due to different trapping behavior of the dyes which changes the influence of electron injection on the majority carrier space charges (see the last paragraph of the discussion). Thus the electrical properties of the polymer matrix and the dye cannot be separated - at least not at these high dye contents.

When investigating the temperature dependence of electroluminescence we found only a slight change of the EL intensity with temperature. Therefore it is reasonable to assume that the injection of electrons takes place by tunneling processes. Table II gives a survey of the characteristic EL data (EL onset field and achievable external quantum efficiency) of all the materials under investigation. The effective barrier heights $\bar{\phi}_{B}$ will be discussed below.

\section{B. Discussion}

In the following the injection and transport of charge carriers in single-layer devices will be discussed and a model will be proposed that enables us to describe brightnessvoltage characteristics. Due to the low hole mobility and traps in PVK ${ }^{13,14}$ the occurence of SCLCs is reasonable. One indication for SCLC is the observed power law for low bias voltages. A stronger argument is the "dephasing" of the current and voltage for small bias. When decreasing the voltage fast enough, e.g., at a rate of $0.1-1 \mathrm{~V} / \mathrm{s}-$ the current changes its sign already for a voltage $V>0 \mathrm{~V}$ and the current can even flow opposite to the external voltage. At zero bias 
there is then still a current flow and the device behaves like a charged battery. This is caused by trapped charges near the injecting contact (space charges). For these reasons we assume that the current is space charge limited at least for thick polymer layers $(d>200 \mathrm{~nm})$.

First of all we should discuss some general properties of SCLCs. One requirement for SCLCs is a contact for carrier injection - holes in our case — which is able to supply more carriers than the bulk material can conduct. Generally, in an analytical treatment of SCLCs it is assumed that this contact is electrically perfect, which implies that the electrical field at the contact vanishes and diffusion currents are negligible. ${ }^{12}$ As a consequence, this leads to an infinite charge carrier density at the injecting contact. Thus the above assumption represents only an idealistic picture which is not strictly valid in a real device near the injecting contact. However, it can be shown that a nonvanishing field at the contact due to a small barrier has only a slight influence on the current-voltage characteristics. For the buildup of space charges it is only necessary that the injection current - part of which flows back into the contact by diffusion - is higher than the current flow across the bulk (see Ref. 15, chapter 9, for an exact treatment of the problem including diffusion and a discussion of the role of the contacts). The use of this simplified theory can also be interpreted as neglect of the contact resistance compared to the bulk resistance. This condition is met in our case for thick polymer layers.

In the following discussion we assume that the electric field vanishes at the hole injecting contact to facilitate the analytical treatment. This makes it easier to derive some general properties concerning the spatial distribution of the electric field inside the device. In this sense, the following results are exact solutions of the simplified theory, however, they should be interpreted with care because the basic assumptions are not strictly valid in a real device. Nevertheless, they present a qualitative picture of the electrical field and potential in the devices under investigation.

In the case of an exponential or Gaussian distribution of the energetic levels of traps or in the trap-free case the $I-V$ characteristics is given by a power law: ${ }^{12}$

$$
I \propto V^{l+1} / d^{2 l+1}
$$

with $l=1$ in the trap-free case and $l>1$ otherwise. With the assumption of a spatially homogeneous trap distribution it is possible to calculate the electrical potential $\phi(x)$ and the electric field $E(x)$ within the device, where $x$ designates the distance to the injecting electrode for majority carriers, i.e., the anode:

$$
\begin{aligned}
& \phi(x)=-V\left(\frac{x}{d}\right)^{(2 l+1) /(l+1)}, \\
& E(x)=\frac{2 l+1}{l+1} \frac{V}{d}\left(\frac{x}{d}\right)^{l /(l+1)} .
\end{aligned}
$$

Figure 5 shows the corresponding curves for the rigid band model and two different SCLC situations. An important consequence of space charges is the fact that at the opposite electrode, the cathode, the electric field is higher than the applied voltage $V$ divided by the polymer thickness $d$ :

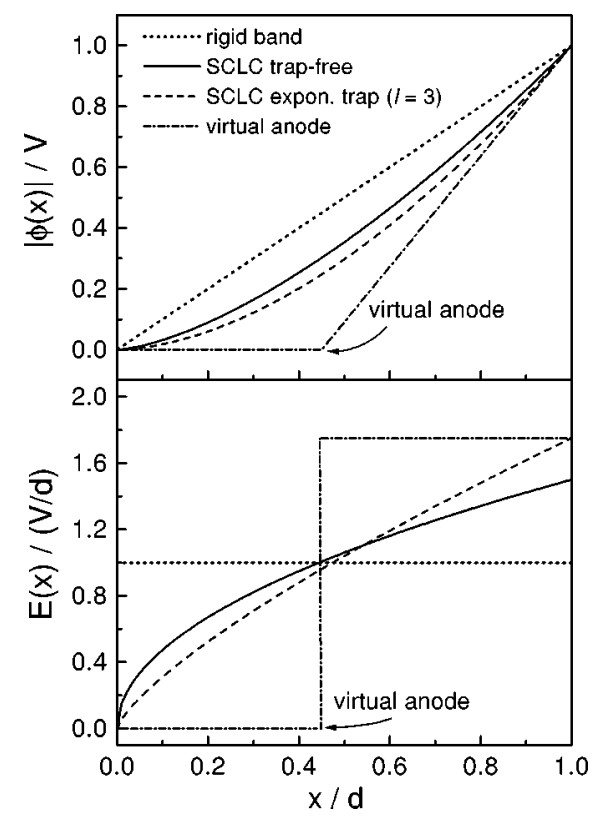

FIG. 5. Electric potential (in units of the applied external voltage $V$ ) and field (in units of the external field $V / d$ ) for the rigid band case and in the presence of SCLC (for the trap-free case and for an exponential trap energy distribution with $l=3$ ). For SCLC the potential and field at the cathode $(x=d)$ take the same values as for a situation with a virtual anode inside the polymer layer (shown here for $l=3$ ).

$$
E(d)=\frac{2 l+1}{l+1} \frac{V}{d}=\gamma \frac{V}{d}>\frac{V}{d} .
$$

The field enhancement factor $\gamma$ takes values in the range of 1.5-2 for the simplified theory. These values should not be taken literally since, even with the knowledge of the exponent $l$ from the power law in the $I-V$ characteristics, $\gamma$ cannot be determined directly because the assumptions are not strictly valid near the interfaces. However, it is obvious that the electric field at the minority carrier injecting electrode will be higher than the "external field" $(V / d)$. This fact has to be taken into account when describing electron tunneling processes in these devices. As shown in Figure 5 the field enhancement at the cathode can be simulated in the rigid band model by a virtual anode within the polymer layer. Figure 6 shows a schematic energy diagram for the above model with the potentials for the electrons (LUMO) and holes (HOMO). Compared to the rigid band case the tunneling distance is reduced by the field enhancement at the cathode caused by SCLC.

Our description of electron injection is based on the following assumptions: As the maximum (external) quantum efficiency of $0.05 \%$ photons per charge carrier is very low, even in the double injection regime the current should be mainly constituted of holes (PVK is known as a hole transport material $\left.^{13}\right)$. Therefore it is reasonable to assume that all the injected electrons recombine while most of the holes pass through the device without recombination. Then the measured light power $P_{E L}$ is proportional to the electron current $I_{e}$. The negligible dependance of the light power on the temperature suggests that the electrons are injected via tunneling at the cathode following a Fowler-Nordheim law ${ }^{12}$ : 


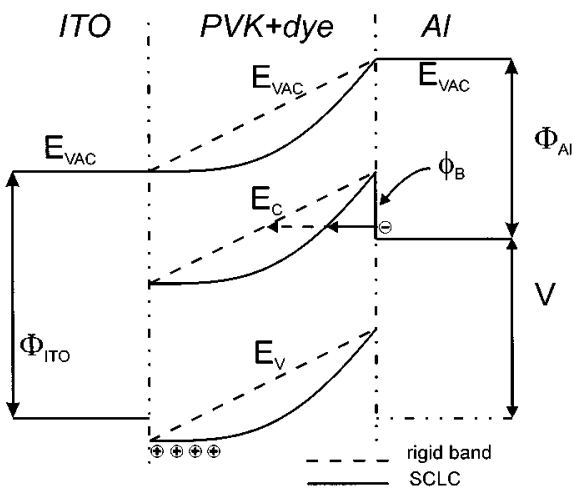

FIG. 6. Schematic energy diagram for the rigid band case (dashed line) and in the presence of space charges (solid line) under forward bias $V$. The anodic space charge leads to a reduction of the electron tunneling distance. Therefore the calculated effective barrier heights $\bar{\phi}_{B}$ are lower than the real barrier height $\phi_{B}$.

$$
P_{E L} \propto I_{e} \propto E^{2} \cdot \exp \left(-\frac{2 \alpha \phi_{B}^{3 / 2}}{3 q E}\right),
$$

where $E$ designates the electric field at the cathode, $\phi_{B}$ the energetic barrier between the Fermi level of the electrode and the LUMO of the organic material, $q$ the elementary charge, and $\alpha=4 \pi \sqrt{2 m^{*}} / h$, with $m^{*}$ usually taken as the free electron mass $m_{0}$. In the case of SCL hole current the electric field at the cathode is $E=\gamma V / d$, with $\gamma>1$. Inserting this relationship into the above equation gives:

$$
P_{E L} \propto V^{2} \cdot \exp \left(-\frac{2 \alpha d \phi_{B}^{3 / 2}}{3 q V \gamma}\right) .
$$

Introducing the external field $\bar{E}=V / d$ and an effective barrier height $\bar{\phi}_{B}=\gamma^{-2 / 3} \phi_{B}\left(\bar{\phi}_{B}<\phi_{B}\right)$ one yields:

$$
P_{E L} \propto \bar{E}^{2} \cdot \exp \left(-\frac{2 \alpha \bar{\phi}_{B}^{3 / 2}}{3 q \bar{E}}\right) .
$$

This equation is of the same form as the Fowler-Nordheim law, however, it does now contain implicitly the measurable quantities $V$ and $d$, while the electric field $E$ in Equation (3) is not directly accessible. The evaluation of Equation (5) is somewhat different than for the conventional FowlerNordheim equation, since from a plot of $\log \left(P_{E L} / \bar{E}^{2}\right)$ vs $1 / \bar{E}$ (analogue to the usual Fowler-Nordheim plot) only the effective barrier height $\bar{\phi}_{B}$ can be determined. The latter has no direct physical meaning but is related to the real barrier height $\phi_{B}$ by means of the field enhancement factor $\gamma$, which is a function of the anodic space charge. $\gamma$ is a priori unknown and must be determined by a different method.

Based on the assumption that $\gamma$ has only a negligible dependence on the dye in the devices under investigation, $\bar{\phi}_{B}$ gives us the possibility of comparing the different dyes qualitatively with regard to the real barrier heights for electron injection. According to Equation (5) a plot of $\log \left(P_{E L} / \bar{E}^{2}\right)$ vs $1 / \bar{E}$ should give a straight line. From the slope the effective barrier height can be determined. In Figure 7 this plot is shown for two different polymer thicknesses. In both cases

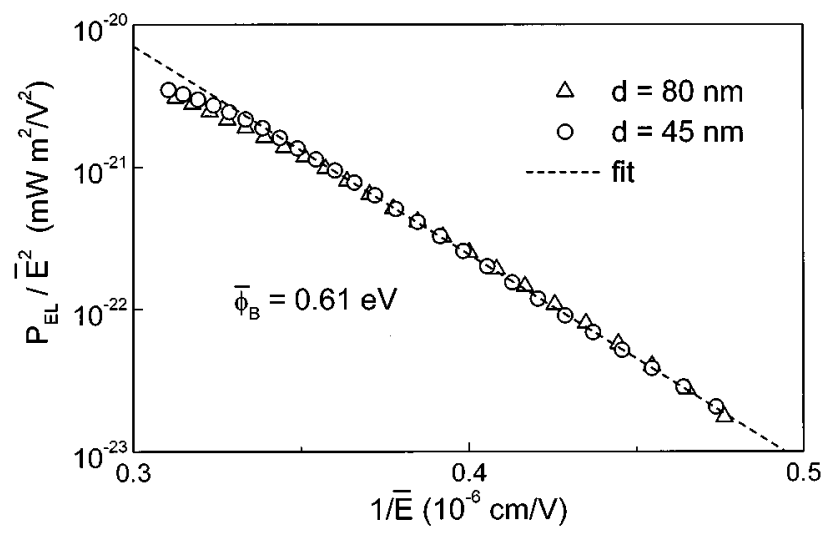

FIG. 7. Fit of the modified Fowler-Nordheim equation (5) to brightnessvoltage characteristics of devices with different thickness, having PVK + dye(4) as emitting layer.

straight lines are obtained which are identical within the error of thickness measurement $(5 \mathrm{~nm})$. With Equation (5) the brightness-voltage characteristics can be described very well over three orders of magnitude in the light power. In Table II the obtained effective barrier heights $\bar{\phi}_{B}$ for the different dyes are given. They are strongly correlated to the electroluminescence onset fields, indicating that the limiting factor for $\mathrm{EL}$ is the electron injection in these devices. The dependence of $\bar{\phi}_{B}$ on the dye suggests that electron injection takes place directly into the LUMO levels of the dye, which act as efffective hole traps. Hence, not only the photoluminescence efficiency but also the LUMO levels of the dyes play an important role for EL - at least for high dye contents.

As we have mentioned before, the steeper increase of the current following the onset of electroluminescence (see Fig. 3) also follows a Fowler-Nordheim behavior. The solid line in Fig. 3 for $V>30 \mathrm{~V}$ indicates the fit to the FowlerNordheim law. In this case the current is much higher than in the case of single carrier injection, although the current is still a majority carrier current. This behavior can only be explained by the influence of electron injection and recombination on the space charge because the simple addition of the electron and the hole current would be approximately equal to the single carrier injection current. Thus the current flow is still bulk-limited. Therefore the Fowler-Nordheim characteristics seen in the current cannot be attributed to an injection limitation via tunneling of the majority carriers (holes).

\section{MULTI-LAYER DEVICES}

\section{A. Experimental results}

The brightness and the external quantum efficiency in the single-layer devices are moderate. However, they can be increased significantly by using additional hole and electron transport layers. As an example we compare in Figure 8 brightness-voltage curves for dye (3) in a single-layer device, a two-layer device with PPV as hole transport material between ITO and the PVK/dye layer and a three-layer device with an additional oxadiazole layer as electron transport material on top of PVK. The inset shows the corresponding 


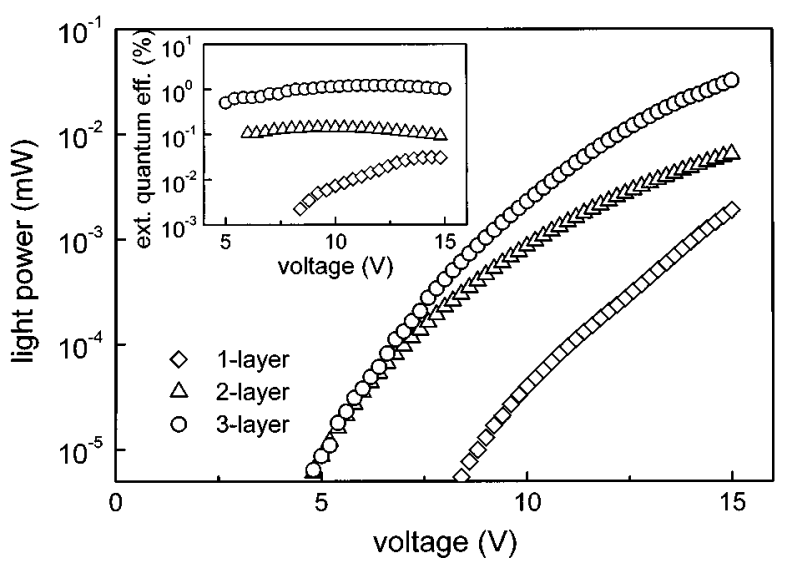

FIG. 8. Comparison of brightness and external quantum efficiency as a function of the applied voltage for single- and multi-layer devices. The single-layer device consisted of a PVK + dye(3) film beetween ITO and Ca electrodes. In the two-layer device PPV was used as additional hole transport layer between ITO and the blend, in the three-layer system an oxadiazole layer was applied as electron transport layer on top of the PVK/dye blend. The respective layer thicknesses are $50 \mathrm{~nm}$ for PPV, $90 \mathrm{~nm}$ for the $\mathrm{PVK} /$ dye blend and $30 \mathrm{~nm}$ for the oxadiazole.

external quantum efficiencies. The introduction of PPV reduces the onset voltage and increases brightness and quantum efficiency. However, for high voltage $(V \approx 15 \mathrm{~V})$ the difference to the single-layer device becomes smaller. An additional oxadiazole layer does not affect the onset voltage but increases brightness and quantum efficiency significantly as compared to the two-layer configuration. At a voltage of $15 \mathrm{~V}$ the luminance reaches $75 \mathrm{~cd} / \mathrm{m}^{2}$ and the external quantum efficiency is around $1 \%$. The maximum achievable luminance in three-layer devices was about $250 \mathrm{~cd} / \mathrm{m}^{2}$. It should be mentioned that due to the high absorption of PPV below $500 \mathrm{~nm}$ its use as a hole transport layer is only possible for materials with emission in the spectral range well above $500 \mathrm{~nm}$. Below, other transparent hole conducting polymers, like e.g., polyaniline or polythiophene, have to be used.

\section{B. Discussion}

The above model cannot be applied easily to multi-layer devices because the field distribution in the different layers is not known a priori. Therefore the discussion for these kind of devices will only be given qualitatively. As we have discussed, in the single-layer device an anodic space charge is formed. This does not necessarily imply a vanishing barrier for hole injection. Since PPV is known to form good hole injecting contacts on ITO $^{16}$ and also has higher conductivity as compared to PVK, one can expect a better hole injection into the PVK/dye layer. This facilitates the formation of the space charge, which in turn leads to a higher field enhancement for electron injection at the cathode. These two effects can explain qualitatively the significant reduction of the EL onset voltage seen in Figure 8. With increasing voltage the improvement of hole injection into the PVK/dye layer in the two-layer device, as compared to the single-layer device, be-
$\underline{E_{\text {vacuum }}}$

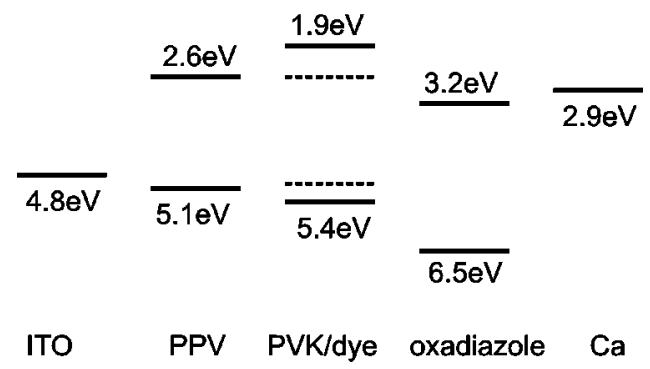

FIG. 9. Schematic energy diagram of the three-layer device composed of the PVK/dye blend with PPV as additional hole transport layer and an oxadiazole as electron transport layer, sandwiched between ITO and Ca electrodes. Energy values of PVK and the oxadiazole are taken from Ref. 7 and 11, respectively. The values of PPV have been determined by ultraviolet photoemission spectroscopy (Ref. 20). The values of the dyes are not known, but should be within the energy gap of PVK.

comes less important, as can be seen from the fact that at 15 $\mathrm{V}$ brightness and efficiency of the single layer device almost reach the values of the two-layer device.

However the quantum efficiency is still not satisfactory because of the imbalanced charge carrier flow (holes are the majority carriers). The latter can be improved by introducing an electron conducting and hole blocking oxadiazole layer. ${ }^{11}$ This can be understood from the schematic energy level diagram of the three-layer device shown in Figure 9. We note that there is considerable uncertainty and variation in the literature data of the energy levels of the materials. Especially, the values of ITO and $\mathrm{Ca}$ (or $\mathrm{Al}$ ) can be influenced by surface contamination or interfacial oxide layers. Nevertheless, one can gain qualitative insight into the relevant device processes. The main effect of the oxadiazole layer is to form a large barrier for holes due to its high HOMO energy, which prevents part of the positive carriers from passing through the device without recombination. Additionally, the LUMO level of the oxadiazole almost matches the work function of $\mathrm{Ca}$, making electron injection into this layer rather easy. Also, the oxadiazole has electron mobilities of $10^{-6} \mathrm{~cm}^{2} / \mathrm{V} \mathrm{s}$ at fields of $7 \times 10^{5} \mathrm{~V} / \mathrm{cm}$ (Ref. 11) - in the same range as the hole mobilities in PVK or PPV. ${ }^{13,17}$ Thus one has to expect an improvement of the charge carrier balance. Another effect of this layer is to move the recombination zone away from the polymer/metal contact where the luminescence is quenched by the presence of nonradiative recombination centres. It has been shown recently on similar materials that within a distance of $10-20 \mathrm{~nm}$ from the metal contact almost all generated excitons are quenched. ${ }^{18}$ With the extremely low electron mobilities in hole conducting polymers like PVK, ${ }^{14}$ the electron penetration depth (assuming an exponential decay of the density of injected electrons in the film due to exciton formation ${ }^{19}$ ) can be in the same range. Therefore, in the single- and two-layer device a large fraction of excitons is quenched nonradiatively at the metal electrode. These drawbacks can be overcome using the additional electron conducting oxadiazole layer which results in significantly improved quantum efficiencies of about $1 \%$. 


\section{CONCLUSION}

Our investigations have shown that the space charges of majority carriers can have an important influence on minority carrier injection even in simple single-layer devices. The space charge of the majority carriers increases the electric field at the opposite electrode leading to a field enhancement for minority carrier injection. As a consequence, in the case of electron injection via tunneling the barrier heights obtained by applying a Fowler-Nordheim law to the brightness-voltage characteristics are only effective values, which are lower than the real barrier heights.

With the high dye concentrations used we observe a dependence of the barrier height on the dye, suggesting that the electron injection takes place directly into the LUMO levels of the dyes. The field enhancement by space charges can be utilised to improve device performance by means of additional hole injection layers together with electron transport layers.

\section{ACKNOWLEDGMENT}

This work has been supported by BMBF/Bayer AG/ Robert Bosch GmbH under Project No. 03N1003A0.

${ }^{1}$ J. Kido, K. Hongawa, K. Okyama, and K. Nagai, Appl. Phys. Lett. 64, 815 (1994).

${ }^{2}$ B. Hu, Z. Yang, and F. E. Karasz, J. Appl. Phys. 76, 2419 (1994).
${ }^{3}$ J. Kido, H. Shionoya, and K. Nagai, Appl. Phys. Lett. 67, 2281 (1995).

${ }^{4}$ C. C. Wu, J. C. Sturm, J. Tian, M. E. Thompson, and R. A. Register, in IEEE International Electron Devices Meeting, Technical Digest, New York, NY, 1995, p. 821.

${ }^{5}$ G. E. Johnson, K. M. McGrane, and M. Stolka, Pure Appl. Chem. 67, 175 (1995).

${ }^{6}$ H. Suzuki and S. Hoshino, J. Appl. Phys. 79, 8816 (1996).

${ }^{7}$ Z. Zhang, X. Jiang, S. Xu, and T. Nagatomo, in Organic Electroluminescent Materials and Devices, edited by S. Miyata and H. S. Nalwa (Gordon and Breach, Amsterdam, 1997).

${ }^{8}$ I. D. Parker, J. Appl. Phys. 75, 1656 (1994).

${ }^{9}$ D. V. Khramtchenkov, H. Bässler, and V. I. Arkhipov, J. Appl. Phys. 79, 9283 (1996)

${ }^{10}$ E. Gautier-Thianche, C. Sentein, J. Nunzi, A. Lorin, C. Denis, and P. Raimond, Synth. Met. 91, 323 (1997).

${ }^{11}$ J. Bettenhausen, P. Strohriegl, W. Brütting, H. Tokuhisa, and T. Tsutsui, J. Appl. Phys. 82, 4957 (1997).

${ }^{12}$ K. C. Kao, and W. Hwang, Electrical Transport in Solids (Pergamon, Oxford, 1981).

${ }^{13}$ J. M. Pearson and M. Stolka, Poly(N-vinylcarbazole) (Gordon and Breach, New York, 1981)

${ }^{14}$ P. M. Borsenberger and D. S. Weiss, Organic Photoreceptors for Imaging Systems (Marcel Dekker, New York, 1993).

${ }^{15}$ M. A. Lampert and P. Mark, Current Injection in Solids (Academic, New York, 1970)

${ }^{16}$ W. Brütting, M. Meier, M. Herold, S. Karg, and M. Schwoerer, Chem. Phys. (in press).

${ }^{17}$ E. Lebedev, T. Dittrich, V. Petrova-Koch, S. Karg, and W. Brütting, Appl. Phys. Lett. 71, 2686 (1997).

${ }^{18}$ J. Grüner, M. Remmers, and D. Neher, Adv. Mater. 9, 964 (1997).

${ }^{19}$ H. Vestweber, H. Bässler, J. Grüner, and R. H. Friend, Chem. Phys. Lett. 256, 37 (1996).

${ }^{20}$ K. Sugiyama, H. Ishii, K. Seki, M. Meier, and W. Brütting (unpublished). 